\section{Optical Experiments to verify the Second Postulate of the Special Theory of Relativity}

KANTOR's experiment ${ }^{1}$, which appeared to contradict the second postulate of special relativity, gave rise to a series of other experiments ${ }^{2-6}$. In all cases these experiments were made with rotating systems, and interpretations of Kantor's experiment have only been made from this point of view ${ }^{7,8}$. Two of these experiments used the same principle but reached different results. Experiment [1] was carried out in air and the magnitude of the fringe shift was $\Delta=0.5$ fringes. In experiment [3], which was carried out first in air, turbulence in the air disturbed the fringes, so that they were extremely unstable. When this experiment was carried out in vacuo, the fringe shift was found to be less than 0.02 fringes. It is therefore interesting to investigate the influence of the air turbulence on the fringe pattern, which we did with a specially adapted Rayleigh interferometer. A diagram of the experimental arrangement is shown in Fig. 1 .

A xenon discharge tube (4) produced short flashes of white light which was collimated and split into two parallel light beams in the interferometer. One of these beams passed through the thin glass window (1) mounted on the rotating arm, while the other passed through a stationary window (2). If the general relativity interpretation of these experiments is correct, wo may consider that the beam belongs to the rotating system after passing through

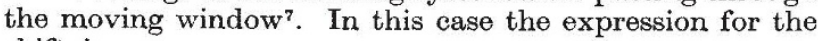
shift is

$$
\Delta=\frac{\omega L r}{c \lambda}
$$

The ballistic theory of light leads to the same result, so that this experiment may also be used to prove the ballistic theory. In our experiment the angular velocity $\omega$ was $314 \mathrm{~s}^{-1}$ (50 rev/sec), the path of light after passing through rotating window, $L$, was $86 \mathrm{~cm}$, the distance from the axis of the rotating arm to the window centre, $r$, was $12.5 \mathrm{~cm}$, and the value taken for wavelength of light, $\lambda$, was $5000 \AA$. Under these conditions, equation (I) gives $\Delta$ as $0 \cdot 22$. Observations were made with the window rotating in both directions, so that the total fringe shift is twice that given by equation (1), that is, $\Delta_{t}=0 \cdot 44$. The thickness of the window was $0.1 \mathrm{~mm}$, so that the shift caused by the dragging effect was negligible.

To ensure that the light beam passed perpendicularly through the rotating window, a mirror (3) was placed at the other end of the rotating arm to reflect the triggering light beam (5) at the photo-cell (6). Electric impulses from the photo-cell were transmitted to the amplifier and stroboscope $(I)$, which provided impulses for the discharged tube (4). Impulses from the amplifier were also led to the counter (II), which was started and stopped at the same

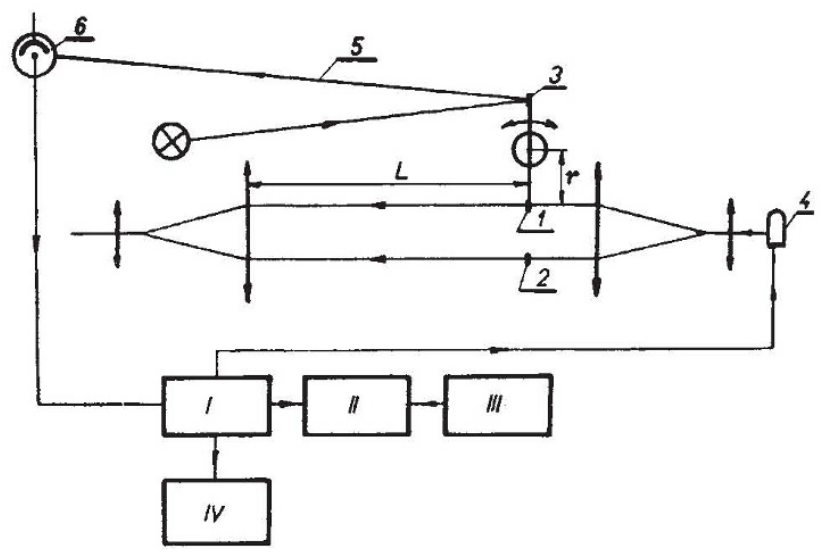

Fig. 1. Diagram of experimental arrangement.

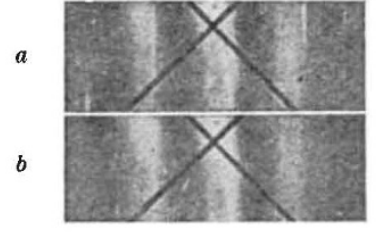

Fig. 2. $a$, Fringes with window rotating at $50 \mathrm{rev} / \mathrm{sec}$ anti-clockwise; $b$, fringes with window rotating at $50 \mathrm{rev} / \mathrm{sec}$ clockwise.

time as the electrical stop watch (III), and the average speed was calculated from the measurements made. The time delay between the reception of the triggering light beam and the flash of the stroboscope was measured by the oscilloscope (IV), and eliminated by shifting the photo-cell up and down.

Observations were made by several independent observers, both visually and photographically. The sensitivity of apparatus was such that a fringe shift of 0.1 fringes would have been observed, but no fringe shift was observed. The fringes photographed with the window rotating at $50 \mathrm{rev} / \mathrm{sec}$ anti-clockwise are shown in Fig. $2 a$, and those photographed with the window rotating at $50 \mathrm{rev} / \mathrm{sec}$ clockwise are shown in Fig. $2 b$. The experiment was made in air and turbulence of the air did not influence the fringe pattern. When streams of warm air were produced in the laboratory, the fringes became unstable and shifted irregularly. It is possible that the shift in experiment [1] and the disturbance of the fringe pattern in air in experiment [3] were caused by the temperature gradient in the air.

We conclude that the result of this experiment is in good agreement with special relativity, which predicts a negligible fringe shift. This experiment, however, does not solve all the problems concerned with the optical verification of the second postulate. It is not clear, for example, that the light source is linked with the rotating frame. This problem cannot be solved by one experiment, but only by a whole series of experiments.

This work has been performed with the co-operation of Dr. V. Vyšín, who suggested the experiment.

\section{J. ZÁHEJSKÝ}

V. Kolesnikov

Department of Experimental Physics,

Palacky University,

Olomouc, Czechoslovakia.

${ }^{1}$ Kantor, W., J. Opt. Soc. Amer., 52, 9, 978 (1962).

' James, J. F., and Sternberg, R. S., Nature, 197, 1192 (1963).

${ }^{3}$ Babcock, G. C., and Bergman, T. G., J. Opt. Soc. Amer., 54, 2, 147 (1964).

'Rotz, F. B., Phys. Lett., 7, 4, 252 (1963).

$\checkmark$ Beckmann, P., Dept. of Elect. Eng., Univ. of Colorado, Reprint (1964)

' Waddoups, R. O., Edwards, W. F., and Merrill, J. J., J. Opt. Soc. Amer., 55, $2,142(1965)$.

'Vyš́n, V., Phys. Lett., 8, 36 (1964).

${ }^{8}$ Datzeff, A. B., C.R. Acad. Sci., Paris, 17, 2, 121 (1964).

\section{THE SOLID STATE}

\section{X-ray Spikes and Metal Inclusions in Synthetic Diamond}

Meyer and Milledge ${ }^{1}$ and Lonsdale and Milledge ${ }^{2}$ reported the presence of anomalous X-ray spikes projecting from the reciprocal lattice points of synthetic diamonds which had been doped with aluminium, boron or nitrogen. Theso spikes were found to differ from those which have been observed in some natural type I diamonds, in that they lay in $\langle 111\rangle$ directions instead of $\langle 100\rangle$ directions, and it was suggested by Lonsdale and Milledge ${ }^{2}$ that the doped synthetic diamonds should show platelets parallel to $\{111\}$ planes. Recently, Phaal and Zuidema ${ }^{3}$ found that most synthetic diamonds contain a 